\title{
CRISPR/Cas9-mediated cleavage of protospacer with DNA damage
}

\author{
I. Vokhtantsev ${ }^{1}$, L. Kulishova ${ }^{2}$, A. Endutkin ${ }^{1,2}$, D. Zharkov ${ }^{1,2 *}$ \\ ${ }^{1}$ Novosibirsk State University, Novosibirsk, Russia \\ ${ }^{2}$ Institute of Chemical Biology and Fundamental Medicine SB RAS, Novosibirsk, Russia \\ *e-mail:dzharkov@niboch.nsc.ru
}

Key words: DNA damage, CRISPR/Cas9 system, Cas9/sgRNA cleavage

Motivation and Aim: A CRISPR/Cas9 system widely uses in the genome editing. The main principle of genome editing is the formation of double-strand breaks in DNA and following DNA reparation. A Cas9/sgRNA complex is targeted to the protospacer in DNA by PAM recognition and R-loop formation. It was shown that mismatches in the sequence of a protospacer influence negatively on the DNA binding and cleavage by the Cas9/sgRNA complex [1]. However, an influence of the DNA damage on cleavage efficiency by the Cas9/sgRNA previously wasn't studied. Thereby we decided to investigate the Cas9/sgRNA cleavage of duplexes and plasmids with point DNA lesions in PAM and the protospacer sequence.

Methods: To detect the DNA cleavage product in vitro, we've obtained radiolabeled duplexes with substitutions such as 8-oxo-2'-deoxyguanosine (oxoG), uridine (U) and tetrahydrofuran abasic site (F). These substitutions contained both in PAM (5'-TGG-3') and the protospacer sequence. In addition, plasmids with the same substitutions as in duplexes have been used as substrates.

Results: Surprisingly, any substitutions in PAM sequence (5'-TGG-3') of guanosine on the tetrahydrofuran abasic site or 8-oxo-2'-deoxyguanosine led to cleavage resistance for duplex substrates. The opposite situation was observed when substrates were plasmids. 8-Oxo-2'-deoxyguanosine in the second and third positions of PAM partially reduced of cleavage efficiency while tetrahydrofuran abasic sites in the same position didn't effect on activity of the Cas9/sgRNA complex. The cleavage efficiency of duplexes with substitutions at the protospacer positions upstream of PAM of non-target strand decreases in the row $(8 \mathrm{G} \rightarrow \mathrm{F})-(8 \mathrm{G} \rightarrow$ oxoG $)-(16 \mathrm{C} \rightarrow \mathrm{U})-(14 \mathrm{C} \rightarrow \mathrm{U})$.

Conclusion: Our results have shown that the DNA cleavage by the Cas $9 / \mathrm{sgRNA}$ complex depends not only on the type of DNA damage, but also on the type of substrate.

\section{References}

1. Zheng T. et al. (2017) Profiling single-guide RNA specificity reveals a mismatch sensitive core sequence. Scientific Reports. 7:40638. 